\title{
The superrotation of solar supergranules
}

\author{
N. Meunier and T. Roudier \\ Laboratoire d'Astrophysique de l'Observatoire Midi-Pyrénées, Université Paul Sabatier, CNRS, 57 Avenue d'Azereix, BP 826, \\ 65008 Tarbes Cedex, France \\ e-mail: [meunier; roudier] @ast.obs-mip.fr
}

Received 22 November 2006 / Accepted 19 January 2007

\begin{abstract}
Context. Supergranules have long been believed to rotate faster than plasma and than magnetic structures. However, it has recently been shown that this could be due to a bias appearing when using Dopplergrams, which are strongly affected by projection effets. Aims. Our purpose is to perform new measurements of supergranule angular velocities using a technique that would not be significantly influenced by projection effets.

Methods. We are therefore tracking horizontal divergence maps smoothed on the supergranular scale, either globally or on the scale of cells. Tracking the magnetic field using the same technique allows them to be used as a reference. Doppler tracking is performed for a direct comparison.

Results. We confirm that the tracking of Doppler features is not reliable when computing the dynamics on the supergranular scale. However, we find that divergence features are still rotating faster than the magnetic field, when using two independent time series. Conclusions. We conclude that the long-standing puzzle of supergranular superrotation persists, and that interpreting it in terms of anchorage depth inside the convective zone may not be sufficient to explain it.
\end{abstract}

Key words. Sun: rotation - Sun: photosphere - Sun: general

\section{Introduction}

Supergranules have been found to rotate significantly faster than plasma, and even faster than the magnetic structures, including the network (Duvall 1980; Snodgrass \& Ulrich 1990). Both these results have been obtained by tracking Doppler features on the solar disk. More recently, Beck \& Schou (2000) found similar results by tracking the features on different scales in Fourier space. More puzzling properties of supergranules were also obtained by Schou (2003), in particular a wavelike behavior of supergranules, although this analysis has been questioned by Rast et al. (2004).

However, very recently, Hathaway et al. (2006) performed a simulation that showed that all these measurements, which were performed using Doppler features, were strongly biased by the projection effects on the observed signal. They concluded that supergranules may not be rotating as fast as previously thought and that the rotation could probably then be explained by the anchoring of supergranules in the convective zone at a depth rotating at the same rate.

It was therefore necessary to measure the angular velocity of supergranules again using a method without any projection effect. We therefore chose to track supergranules using horizontal divergence maps as determined by Meunier et al. (2007a,b,c). We used the same data sets as in Meunier et al. (2007a,b) while Meunier et al. (2007c) used only a subset. Tracking magnetograms is used for reference, and Doppler features are tracked for comparison. We describe the data in Sect. 2. The global tracking is presented in Sect. 3 and the feature tracking in Sect. 4. We discuss the results in Sect. 5.

\section{Data and processing}

\subsection{MDI data}

We used high resolution MDI/SOHO (Scherrer et al. 1995) intensity maps, magnetograms, and Dopplergrams obtained during the solar minimum in January 1997 and March 1997. The first series started on January 16, 1997 at 23:00 and lasted $46 \mathrm{~h}$. The second series started on March 17, 1997 at 11:00 and lasted $15 \mathrm{~h}$. A few images were missing in each time series, typically one or two images per hour. They were replaced by interpolating between adjacent images. The pixel size is 0.605 arcsec and the field-of-view $620^{\prime \prime} \times 303^{\prime \prime}$. The temporal cadence is $1 \mathrm{~min}$. All images are remapped on a grid with constant steps in longitude and latitude.

\subsection{Divergence maps}

For each of the 61 hourly data sets, the 60 intensity maps and magnetograms were aligned with the map at the center of the data set. Intensity maps were corrected for large-scale gradients and $\mathrm{k}-\omega$ filtered (with a cut-off at $6 \mathrm{~km} \mathrm{~s}^{-1}$ ). A local correlation tracking (LCT) algorithm was then applied to provide velocity and divergence maps. These divergence maps are averaged over $1 \mathrm{~h}$ and smoothed with a Gaussian whose full-width at halfmaximum is $10.2 \mathrm{Mm}$ to exhibit supergranular cells. The validity of this approach is discussed in Meunier et al. (2007b,c). The displacements between maps separated by a given time-lag $(1 \mathrm{~h}$ or more) will be computed using several techniques described in Sect. 3. 


\subsection{Magnetogram reference}

It is very important to compare the angular velocity derived from horizontal divergence maps with that obtained with other variables, such as the magnetic field, using the same technique, because there is a large dispersion of the magnetic structure's angular velocity in the literature. Here we consider the 1-h averaged magnetograms, with a low noise level $(\sim 3 \mathrm{G})$. These maps can be used exactly in the same way as the divergence maps, and the magnetic network angular velocity will be used as a reference. Note that the magnetic network is located in regions of strong converging flows as expected (Meunier et al. 2007a,b). A pore with active network surrounding it is in the field-of-view. Most of the large boxes used in Sect. 3 do not include it, however, except at the end of the sequence (but see discussion in Sect. 3.1), and cells close to the pore have been eliminated in Sect. 4.

\subsection{Doppler maps}

Because the previous results using Dopplergrams have been questionned by Hathaway et al. (2006), it was interesting to compare the displacements obtained in the same conditions for Dopplergrams. We therefore also consider 1-h averaged Dopplergrams, from which the fundamental rotation signal has been removed after an estimation using a linear fit for each line of the images. In this resulting Dopplergram, small-scale structures are still visible, as can be confirmed by comparing the Fourier transform of these images with the smoothed divergence maps used above. To deal with features on the same scale as the divergence maps of Sect. 2.2 (supergranulation), we spatially smooth these Dopplergrams with a Gaussian whose half-width at half-maximum is $\sim 7 \mathrm{Mm}$, to obtain a spatial Fourier transform similar to the one in the divergence maps. The structures are also visually similar (except for the strong projection effects in Dopplergrams of course), as shown in Fig. 1. The resulting maps still contain some very large-scale variations due to the projection effects. Finally, it is interesting to note that the computation of the displacement between consecutive maps for various values of the spatial smoothing (from no smoothing to more than $10 \mathrm{Mm}$ ) shows that it is very sensitive to the smoothing and reaches a maximum around $5 \mathrm{Mm}$. This is probably due to the strong projection effects. We show in Sect. 3.2 that the angular velocity is strongly affected by these projection effects.

\section{Global correlation tracking}

\subsection{Divergence map tracking}

We first compute global displacements between two subsequent magnetograms or divergence maps by correlation. We consider square boxes $\left(401 \times 401\right.$ pixels, i.e. $\left.14 \times 14^{\circ 2}\right)$ centered on disk center. An example of the three kinds of maps is shown in Fig. 1. To illustrate the behavior of each type of data, Fig. 2 shows a cut in the longitude direction for a given latitude, and follows this cut in time for the January 1997 time series. The global magnetic field displacement is taken as the reference and removed in this figure. We can observe very long-lived divergence features, as already observed by DeRosa \& Toomre (2004) for example, as well as long-lived network structures.

Table 1 shows the results for a time lag of $1 \mathrm{~h}$ for the two time series. The maximum correlation is smaller for the divergence maps ( 0.68 and 0.67 for the two time series) than for the magnetic field (0.93 and 0.82), indicating a stronger distortion on short time scales for horizontal divergences. In all cases, the
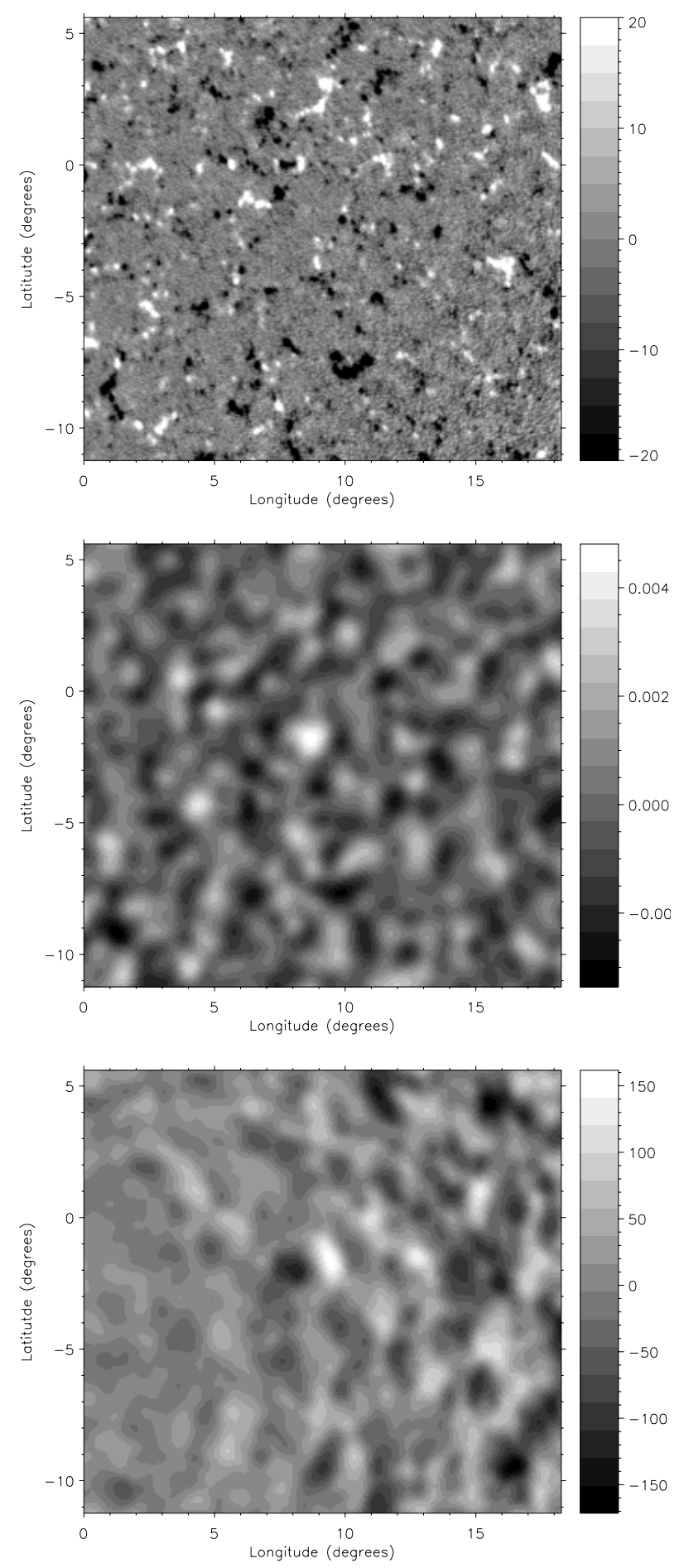

Fig. 1. Upper panel: magnetogram averaged over $1 \mathrm{~h}$, after remapping, showing the line-of-sight magnetic field (in Gauss). Only half of the available field-of-view is shown. Middle panel: same for the horizontal divergence at the supergranular scale (in $\mathrm{s}^{-1}$ ). Lower panel: same for the Dopplergram (line-of-sight velocity field in $\mathrm{m} / \mathrm{s}$ ), after angular correction and spatial smoothing (see text).

amplitude of these correlations is sufficient for computing precise displacements. We therefore obtain a larger angular velocity for divergence maps compared to magnetograms, with a difference of $0.17 \pm 0.08 \mathrm{deg} /$ day for the January 1997 time series and of $0.35 \pm 0.11 \mathrm{deg} / \mathrm{day}$ for the March 1997 time series, which is significantly different from zero at the $1 \sigma$ level $(2 \sigma$ level in the case of the March time series). The amplitude of the difference 

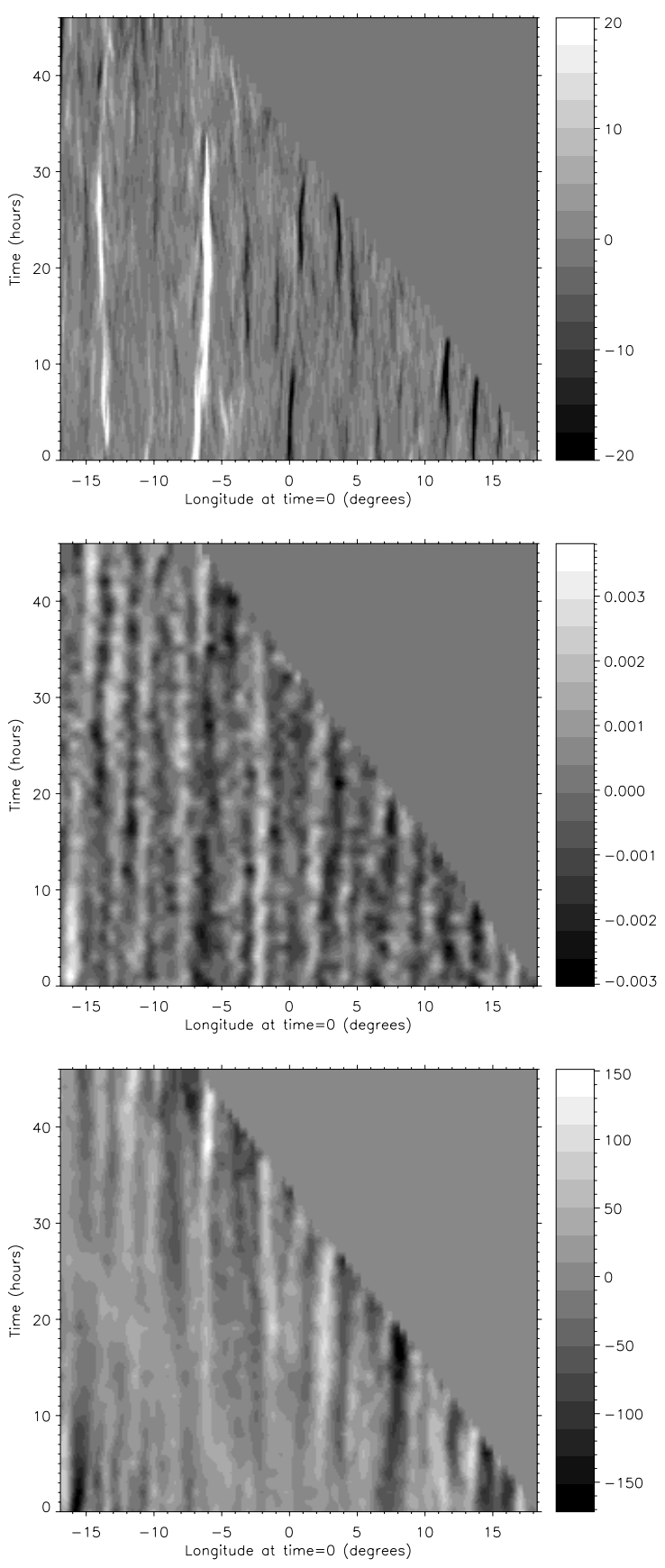

Fig. 2. Upper panel: line-of-sight magnetic field (in Gauss) versus longitude and time, after an averaging over $0.4^{\circ}$ in latitude, for latitude $-2.8^{\circ}$ (crossing disk center). Middle panel: same for the horizontal divergence $\left(\mathrm{in} \mathrm{s}^{-1}\right.$ ) on the supergranular scale. Lower panel: same for the Dopplergram (line-of-sight velocity in $\mathrm{m} / \mathrm{s}$ ), after angular correction and spatial smoothing (see text).

between the divergence map shift and the Carrington rotation is on the order of $36 \mathrm{~m} / \mathrm{s}$ for the January time series, and very similar for the March time series, i.e. a factor of two smaller than the results of Lisle et al. (2004). A small variation with longitude is observed, as in previous works (e.g. Meunier 1999).

Despite coming to the same conclusion for the two data sets, i.e. a higher angular velocity for the divergence maps compared to the magnetic-field, there are few differences that are worth pointing out. The difference in the residual is mostly due to a magnetic field angular velocity variation, weaker in the case of

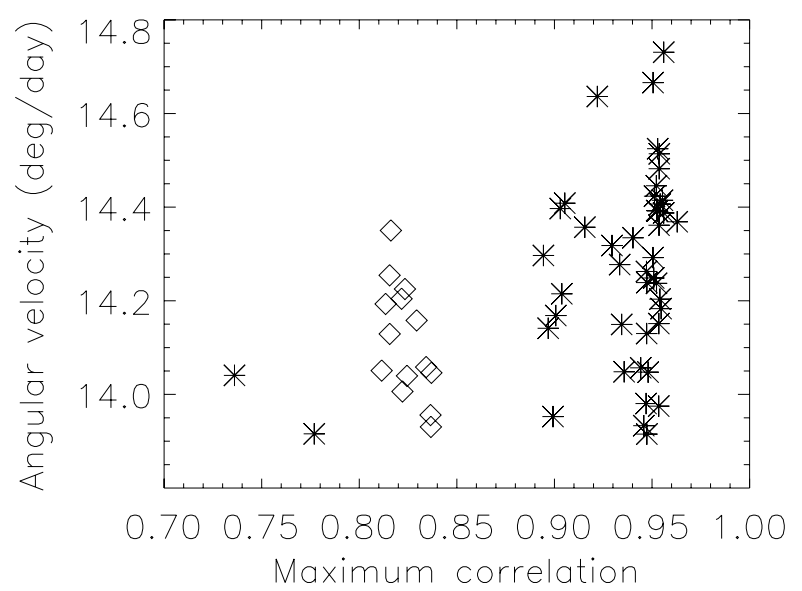

Fig. 3. Sidereal rotation rate derived from the global tracking of the magnetic field versus the maximum correlation between magnetograms, for January 1997 (stars) and March 1997 (diamonds).

the March 1997 time series. The origin of this discrepancy, as well as the difference in maximum correlation shown in Table 1, is illustrated in Fig. 3. The January 1997 time series exhibits a larger maximum correlation, which is due to the presence of the pore, except for the two first images, for which the pore was not present (corresponding to the two points in the lower left corner of the figure). The March 1997 time series exhibit an intermediate maximum correlation, likely to be due to an intermediate activity level. Therefore the higher angular velocity for the January time series is likely to be due to the presence of that pore, showing a significant variation in the angular velocity with the activity level, as already observed in previous works (see the discussion in the next section). However, the angular velocity derived from the divergence maps remains stable and the residual always has a positive sign.

To take the small differential rotation into account (expected to be of the order of 0.1 pixel given our pixel size and fieldof-view), we also computed the correlation for latitude strips of 1 degree. The results are very similar, even when considering a larger field-of-view, i.e. for strips covering the whole longitude range. It also shows that the presence of the pore at the end of the sequence, in the $401 \times 401$ pixel box, does not affect the result much, as the residual angular velocities are similar at latitudes. In this case, the larger maximum correlation for the strip containing the pore is also observed, however, the variation in the angular velocity with the correlation is not significant.

When using different time lags, a small variation in angular velocity is observed, but is not very significant compared to the uncertainties on the measurements. However, the difference between the two types of structures, magnetic fields and divergences, remains similar. Our result therefore seems very robust.

\subsection{Comparison with Doppler map tracking}

In the case of Dopplergrams, surprisingly, we do not observe that the Doppler features are rotating faster than the magnetic field. On the contrary, they rotate slower, the residuals with respect to the magnetic-field angular velocity being $-0.23 \pm 0.04 \mathrm{deg} / \mathrm{day}$ for the January time series and $-0.24 \pm 0.08 \mathrm{deg} / \mathrm{day}$ for the March time series. Furthermore, we observe that the displacement computation is very sensitive to the size of the box and to the spatial smoothing, which is probably for the same reason as the bias discussed by Hathaway et al. (2006), i.e. strong 
Table 1. Sidereal angular velocity (in deg/day) derived from the global tracking for magnetic field $\left(\Omega_{\mathrm{B}}\right)$, for horizontal divergence maps $\left(\Omega_{\mathrm{div}}\right)$, and for smoothed Dopplergrams $\left(\Omega_{\mathrm{dop}}\right)$.

\begin{tabular}{|c|c|c|c|c|c|c|c|c|c|}
\hline Series & \# sets & $\Omega_{\mathrm{B}}$ & $C_{\mathrm{B}}$ & $\Omega_{\text {div }}$ & $\Delta \Omega_{\text {div }}$ & $C_{\mathrm{div}}$ & $\Omega_{\text {dop }}$ & $\Delta \Omega_{\text {dop }}$ & $C_{\text {dop }}$ \\
\hline January 1997 & 46 & $14.27 \pm 0.03$ & 0.93 & $14.44 \pm 0.08$ & $0.17 \pm 0.08$ & 0.68 & $14.04 \pm 0.04$ & $-0.23 \pm 0.05$ & 0.92 \\
\hline March 1997 & 15 & $14.11 \pm 0.03$ & 0.82 & $14.46 \pm 0.11$ & $0.35 \pm 0.11$ & 0.67 & $13.87 \pm 0.07$ & $-0.24 \pm 0.08$ & 0.88 \\
\hline
\end{tabular}

projection effects that are absent from the horizontal divergence maps. For example, when changing the size of the box from $101^{2}$ to $401^{2}$ pixels, the angular velocity of the magnetic field and divergence maps remain within their errorbars, while the angular velocity derived from Dopplergrams varies from $13.45 \pm 0.20 \mathrm{deg} / \mathrm{day}$ to $14.04 \pm 0.04 \mathrm{deg} / \mathrm{day}$ in the January time series. It is also much more variable with longitude. This shows that the Dopplergrams are not very reliable when one wishes to compute the dynamics of supergranules.

\section{Horizontal divergence feature tracking}

To complete our analysis, we also performed a local tracking, centered on either the region of maximum divergence (diverging flows) or on the region of minimum divergence (converging flows). Here we focus on the diverging flows. This approach is therefore similar to a local correlation tracking (we consider a box of size $65 \times 65$ pixels, i.e. $\sim 2.3 \times 2.3$ degrees or $28 \times 28 \mathrm{Mm}^{2}$ ), but is also close to a feature tracking, as the velocities are computed only at the position of supergranular cells. The size of the box is close to the supergranular's typical size. For each structure identified by the position of maximum divergence, for example, we also consider the value of this divergence, the magnetic field level. The magnetic level was computed using two different approaches (all on the absolute value): 1) average for all pixels in the box, 2) for pixels with magnetic fields larger than $10 \mathrm{G}$ (typically network magnetic fields).

For a time lag of $1 \mathrm{~h}$, a summary of the results is shown in Table 2. The average maximum correlation is indicated (respectively $C_{\text {mag }}$ and $C_{\text {div }}$ ), as well as the residual angular velocity (in $\mathrm{deg} /$ day) after substraction of the magnetic angular velocity for horizontal divergence maps $\left(\Delta \Omega_{\text {div }}\right)$. The results are shown for diverging flows (Div) and for converging flows (Conv). The average maximum correlation is indicated $\left(C_{\mathrm{mag}}, C_{\mathrm{div}}\right.$, and $\left.C_{\mathrm{dop}}\right)$, as well as the residual angular velocity (in deg/day) after substraction of the magnetic angular velocity for horizontal divergence maps $\left(\Delta \Omega_{\text {div }}\right)$ and for smoothed Dopplergrams $\left(\Delta \Omega_{\text {dop }}\right)$. We consider 4979 boxes, or "cells", for the January 1997 time series, and 1622 cells for the March 1997 time series. Each of these cells is associated to a local maximum in the divergence map (and therefore to a supergranular cell). The difference in angular velocities between the magnetic field and the divergences is positive in both cases; i.e. the divergence features are again rotating faster than the magnetic field. As for the global tracking, we observe higher angular velocity for diverging flows compared to the magnetic network. The average maximum correlation is 0.73 , i.e. slightly larger than for the global tracking, and the peak of the distribution of the maximum correlation is even higher (close to 0.80). The amplitude of the difference agrees with the result of the previous section, within the errorbars. We have not observed any significant variations in these residuals with the amplitude of the maximum divergence (which is correlated with the size of the cell, Meunier et al. 2007c) nor with the maximum correlation.

However, as for the global tracking, most of the difference comes from the variation in the angular velocity of the magnetic field, as illustrated in Fig. 4. In all cases strong fields are better correlated than weak fields, and are associated to higher angular velocities. The slope between the angular velocity and the magnetic field averaged over the cell is $0.0047 \pm 0.0008 \mathrm{deg} / \mathrm{day} / \mathrm{G}$, which corresponds to a difference of $0.24 \pm 0.04 \mathrm{deg} / \mathrm{day}$ between very weak fields and a $50 \mathrm{G}$ field. The slope between the angular velocity and the maximum correlation is also very significant, with values of $0.83 \pm 0.08 \mathrm{deg} / \mathrm{day}$ and $0.64 \pm 0.18 \mathrm{deg} / \mathrm{day}$ respectively for the January 1997 and the March 1997 time series. The increase in angular velocity with increasing magnetic field inside the cell is in agreement with the results of Meunier (2005a).

When considering the average magnetic field in the box for fields larger than $10 \mathrm{G}$ (i.e. corresponding mostly to the network, case \#2 above), the slope of the residuals versus the magnetic field is significantly negative at the $1 \sigma$ level $(-0.02 \pm$ $0.01 \mathrm{deg} / \mathrm{day} / \mathrm{G})$, for the January 1997 time series, indicating a larger residual for weaker magnetic network. The slope is smaller, $-0.008 \pm 0.004 \mathrm{deg} / \mathrm{day} / \mathrm{G}$, when considering all magnetic field values (case \#1 above). However, the slopes for the March 1997 time series, despite having the same sign, are not significantly different from zero, due to the larger errorbars. This may be due to the the smaller range in magnetic fields in this quieter data set, which is illustrated in Fig. 4. By following each cell during its lifetime, we also determined that long-lived cells had a higher angular velocity. This should be studied further using a larger data set, as the uncertainties remain large. However, it may contradict the results of DeRosa \& Toomre (2004), who found cells with longer life in a more active network, which they interpreted as a possible stabilization effect of the magnetic field on the cells.

The dispersion of the difference between the diverging flows and the magnetic field angular velocities is quite large, on the order of several deg/day, despite the small average residual. The illustration in Fig. 2 seems to indicate that this large dispersion is not due to uncertainties in the measurements but to actual drifts of the cells in one direction or another. Whether these drifts are random or are clues of systematic large-scale flows is the subject of future work.

When we increase the time lag, the angular velocity of diverences decreases. For long time lags, the selection is biased toward long-lived cells, which may have a higher angular velocity, so this does not agree with the observed variation with the time lag. The variation with the time lag may therefore be related to residual projection effects.

Finally, it should be pointed out that the computation performed on converging flows (and therefore selection location of minimum divergence), which is quite independent, provide very similar results, with a residual of $0.30 \pm 0.06 \mathrm{deg} / \mathrm{day}$ (Table 2).

\section{Conclusion and discussion}

We have compared the angular velocity of supergranules defined by horizontal divergences with that of magnetic structures and Doppler features with similar techniques, either a global tracking or a local tracking. In all cases and for the two independent time 
Table 2. Sidereal angular velocity (in deg/day) derived from the local tracking for magnetic field $\left(\Omega_{\mathrm{B}}\right)$ and for horizontal divergence maps $\left(\Omega_{\mathrm{div}}\right)$.

\begin{tabular}{lccccccc}
\hline \hline Serie & Type & $\#$ cells & $\Omega_{\mathrm{B}}$ & $C_{\mathrm{B}}$ & $\Omega_{\text {div }}$ & $\Delta \Omega_{\text {div }}$ & $C_{\text {div }}$ \\
\hline January 1997 & Div & 4979 & $14.18 \pm 0.01$ & 0.79 & $14.35 \pm 0.07$ & $0.26 \pm 0.07$ & 0.73 \\
March 1997 & Div & 1622 & $14.02 \pm 0.02$ & 0.72 & $14.40 \pm 0.13$ & $0.47 \pm 0.13$ & 0.73 \\
January 1997 & Conv & 5327 & $14.19 \pm 0.01$ & 0.80 & $14.38 \pm 0.07$ & $0.30 \pm 0.06$ & 0.73 \\
March 1997 & Conv & 1775 & $14.03 \pm 0.02$ & 0.73 & $14.67 \pm 0.12$ & $0.61 \pm 0.12$ & 0.73 \\
\hline
\end{tabular}
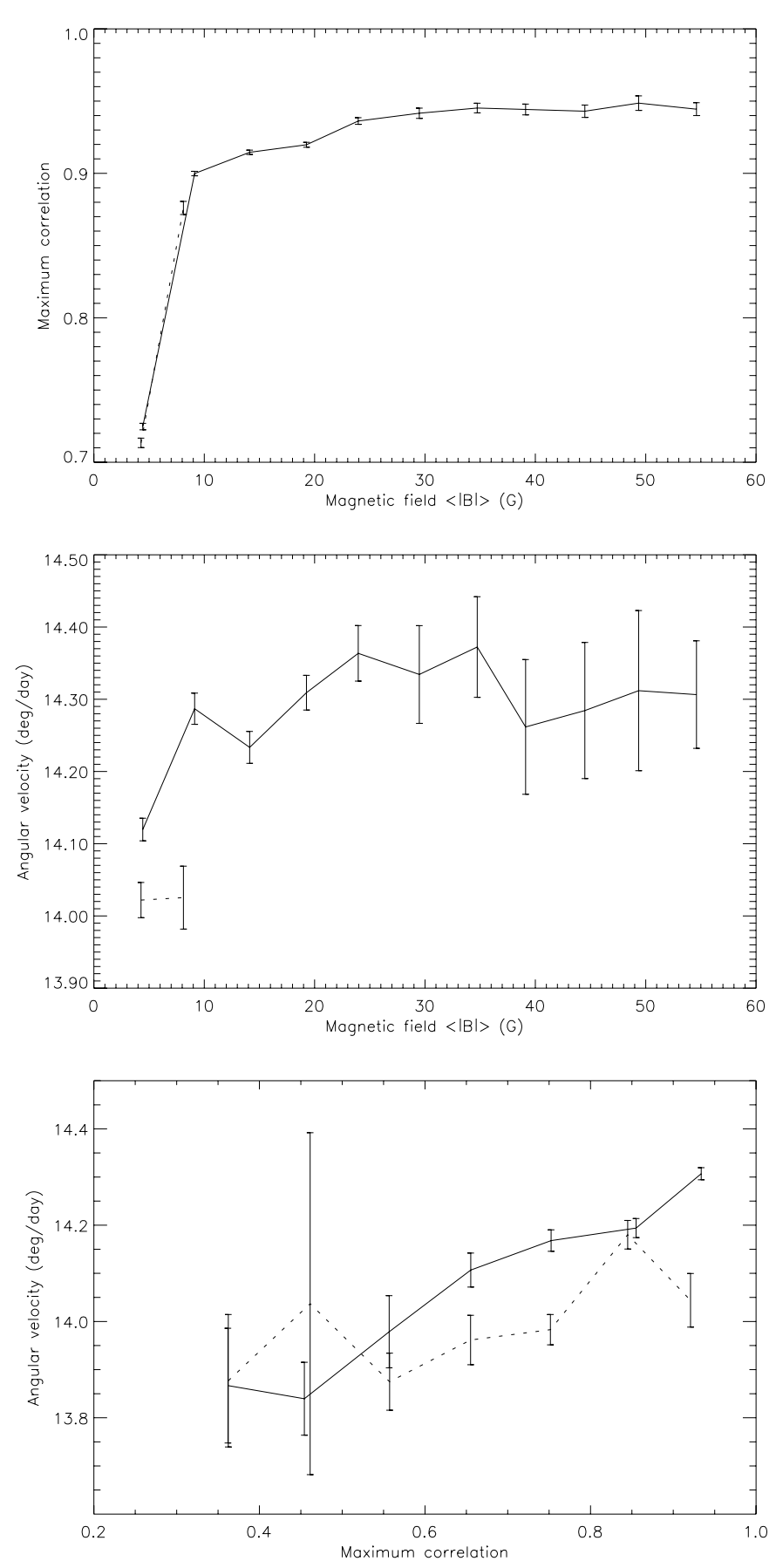

Fig. 4. Upper panel: maximum correlation versus the magnetic field averaged over the cells, derived from the feature tracking of diverging flows, for the January 1997 series (solid line) and for the March 1997 series (dotted line). Most of the signal above $10 \mathrm{G}$ is due to the presence of a pore surrounded by active network in the January 1997 series. Middle panel: same for the sidereal rotation rate derived from the magnetograms versus the magnetic field averaged over the cells. Lower panel: same for the sidereal rotation rate derived from the magnetograms versus the maximum correlation. series, we observe that supergranules do rotate faster than the magnetic field. The results are signicant at the $1 \sigma$ level in the case of the global tracking and at the $3 \sigma$ level in the case of the local tracking. Because we used the same data to compute the magnetic field angular velocity used as a reference, and not the published values, the residuals are free of the influence of temporal variations in the angular velocity.

Comparing the absolute values of the angular velocities is not easy, as magnetic tracers have been shown to exhibit a variable angular velocity during the solar cycle or with the properties of the structures (such as their size). This is the case for sunspots (Balthasar \& Wöhl 1980; Gilman \& Howard 1984; Balthasar et al. 1986; Lustig \& Hanslmeier 1987; Hathaway \& Wilson 1990; Nesme-Ribes et al. 1993; Nesme-Ribes et al. 1997; Brajša et al. 2006) or for the magnetic network (Komm et al. 1993; Meunier et al. 1997; Meunier 2005b) for example. Most of these works show a higher angular velocity at the time of maximum. The variation of the supergranular rotation rate has not been studied in the past, however. Here, we do find a velocity that is significantly lower than the rates derived by Duvall (1980); Snodgrass \& Ulrich (1990). In the first case, this may be explained by the observations of Duvall (1980) done at a time having been of high activity, while we are using observations obtained during the cycle minimum. The difference with the results of Snodgrass \& Ulrich (1990) may be due to the same reason, as they are averaged over approximately two solar cycles and therefore correspond to a higher activity level on average. The differences could also be due to the presence of large-scale patterns of velocities, like those described by Schroeter \& Wöhl (1976) for example. This would not apply to the results of Snodgrass \& Ulrich (1990), as they cover a very long period, but may apply to ours or to the results of Duvall (1980). Furthermore, the rate derived for boxes covering supergranules agrees well with the angular velocity obtained by Meunier (2005b) for 1997 using a similar method on full-disk magnetograms for the same period (1997). The results of Meunier (2005b) were averaged over the whole year and a more detailed analysis would be necessary to compare the absolute values of the angular velocities more precisely. However, the angular velocity of divergences determined globally is intermediate between this angular velocity and the one determined using small magnetic feature tracking. As discussed in Meunier (2005a), these angular velocities are too high to be explained by a simple anchoring depth of structures.

The angular velocity derived from Dopplergrams was found to be much more sensitive to the conditions (size of the box, time lag, smoothing) and to be in general smaller than the magnetic field angular velocity. This is surprising as the simulation performed by Hathaway et al. (2006) show that they should be overestimated due to the projection effects. It can also be pointed out that MDI velocity fields may be slightly underestimated, as shown by Wöhl \& Schmidt (2000). However, this would bias the angular velocity of the plasma derived from the amplitude of the line-of-sight velocity, but not necessarily the angular velocity of the structures identified in the velocity field itself. It is also 
well-known that the local magnetic field may influence the estimation of the line-of-sight velocity. However, here again, as we are only considering the velocity patterns, it is likely that the projection effects are dominating the measurement of the amplitude of the velocity structure angular velocity.

Acknowledgements. SOHO is a mission of international cooperation between the European Space Agency (ESA) and NASA. We thank the referee for suggestions that helped to improve the paper.

\section{References}

Balthasar, H., \& Wöhl, H. 1980, A\&A, 92, 111

Balthasar, H., Vazquez, M., \& Wöhl, H. 1986, A\&A, 155, 87

Beck, J. G., \& Schou, J. 2000, Sol. Phys., 193, 333

Brajša, R., Ruždjak, D., \& Wöhl, H. 2006, Sol. Phys., 237, 365

DeRosa, M. L., \& Toomre, J. 2004, ApJ, 616, 1242

Duvall, Jr., T. L. 1980, Sol. Phys., 66, 213

Gilman, P. A., \& Howard, R. 1984, ApJ, 283, 385
Hathaway, D. H., Williams, P. E., \& Cuntz, M. 2006, ApJ, 644, 598 Hathaway, D. H., \& Wilson, R. M. 1990, ApJ, 357, 271

Komm, R. W., Howard, R. F., \& Harvey, J. W. 1993, Sol. Phys., 145, 1

Lisle, J. P., Rast, M. P., \& Toomre, J. 2004, ApJ, 608, 1167

Lustig, G., \& Hanslmeier, A. 1987, A\&A, 172, 332

Meunier, N. 1999, ApJ, 527, 967

Meunier, N. 2005a, A\&A, 436, 1075

Meunier, N. 2005b, A\&A, 442, 693

Meunier, N., Nesme-Ribes, E., \& Grosso, N. 1997, A\&A, 319, 673

Meunier, N., Roudier, T., \& Tkaczuk, R. 2007a, A\&A, in press

Meunier, N., Tkaczuk, R., \& Roudier, T. 2007b, A\&A, 463, 745

Meunier, N., Tkaczuk, R., Roudier, T., \& Rieutord, M. 2007c, A\&A, 461, 1141

Nesme-Ribes E., Ferreira E. \& Mein P. 1993, A\&A, 274, 563

Nesme-Ribes, E., Meunier, N., \& Vince, I. 1997, A\&A, 321, 323

Rast, M. P., Lisle, J. P., \& Toomre, J. 2004, ApJ, 608, 1156

Scherrer, P. H., Bogart, R. S., Bush, R. I., et al. 1995, Sol. Phys., 162, 129

Schou, J. 2003, ApJ, 596, L259

Schroeter, E. H., \& Wöhl, H. 1976, Sol. Phys., 49, 19

Snodgrass, H. B., \& Ulrich, R. K. 1990, ApJ, 351, 309

Wöhl, H., \& Schmidt, W. 2000, A\&A, 357, 763 